\title{
Frequency resolved wavefront retrieval and dynamics of diffractive focused ultrashort pulses
}

\author{
Benjamín Alonso, ${ }^{1, *}$ Rocío Borrego-Varillas, ${ }^{1}$ Omel Mendoza-Yero, ${ }^{2,3}$ Íñigo J. Sola, ${ }^{1}$ Julio San Román, ${ }^{1}$ \\ Gladys Mínguez-Vega, ${ }^{2,3}$ and Luis Roso ${ }^{4}$ \\ ${ }^{1}$ Universidad de Salamanca, Grupo de Investigación en Óptica Extrema (GIOE) Pl. de la Merced s/n E-37008 \\ Salamanca, Spain \\ ${ }^{2}$ GROC UJI, Departament de Física, Universitat Jaume I, E12080 Castelló, Spain \\ ${ }^{3}$ Institut de Noves Tecnologies de la Imatge (INIT), Universitat Jaume I, E 12080 Castelló, Spain \\ ${ }^{4}$ Centro de Láseres Pulsados, CLPU, E-37008 Salamanca, Spain \\ ${ }^{*}$ Corresponding author: b.alonso@usal.es
}

Received April 10, 2012; accepted June 1, 2012;

posted June 18, 2012 (Doc. ID 166298); published July 17, 2012

\begin{abstract}
In this work we demonstrate the ability of the spatiotemporal characterization technique STARFISH to retrieve the wavelength dependent wavefront of focused ultrashort laser pulses. The high resolution achievable with this technique allows measuring the wavefront at the focal spot. In particular, the method is applied to study the effects of focusing with a kinoform diffractive lens. The evolution from converging to diverging wavefronts as the pulse propagates along the focal region is analyzed for each wavelength. The spatiotemporal intensity and spatially resolved spectrum structure of the pulses, as well as their profiles on axis, are also presented. Numerical simulations of the propagation of such pulses confirm the experimental results. (c) 2012 Optical Society of America OCIS codes: $\quad 050.1965,050.1970,320.7100,010.7350$.
\end{abstract}

\section{INTRODUCTION}

The detailed knowledge of the spatial and temporal structure of light pulses is relevant for pulse control in their applications. Currently, to characterize the temporal structure (amplitude and phase) of the electric field of ultrashort pulses several techniques have been successfully developed [1] . Nevertheless, the need of further information of the pulses led to the implementation of new techniques for the characterization of the spatiotemporal dependent amplitude and phase of the pulses. These are the cases of the spatially encoded arrangement temporal analysis by dispersing a pair of light electric-fields (SEA TADPOLE) [2], shackled-FROG [3] or the spatiotemporal amplitude-and-phase reconstruction by Fourier-transform of interference spectra of highcomplex-beams (STARFISH) [4].

For many applications (e.g., optics aberrations or processes that require phase-matching) it is also necessary to know the wavefront of the pulses. However, standard techniques for this purpose, such as Hartmann-Shack (H-S) [5] and multiwave shearing interferometry [6], present disadvantages for wavefront sensing of ultrashort pulses, especially after diffractive elements. Firstly, due to the spatial resolution limit, these methods cannot measure the wavefront in the vicinity of the focus. For example, in the case of H-S sensors, the size of the microlenses imposes a limit in the resolution. To the best of our knowledge, the smaller commercially available is $30 \mu \mathrm{m}$, which is clearly not enough to characterize the wavefront in the focus. In the case of shearing interferometers it is possible to achieve a higher resolution, but it is limited by the pixel size of the CCD, which usually makes them inappropriate for measurements in the focus. Since STARFISH is a fiber-based device, these problems are avoided achieving resolutions in the order of the fiber core. Second, they measure the averaged wavefront for the whole ultrashort pulse, neglecting the obvious wavelength dependence. This problem can be solved, e.g., by using interference filters [7] to select certain wavelengths of the pulse, thus obtaining only some slices of the wavefront with a precision limited by the filter quality. In another approach, the second-harmonic generation (SHG) is used for wavefront retrieval, since the conversion efficiency of this process strongly depends on the wavelength and the angle between the wave-vectors and the optical axis [8]. In this case, the error in the retrieval may be large under strongly focusing conditions, since noncollinear processes may play an important role. Another diagnostic related to this topic consists in combining a SHG-based wavefront measurement with a spatially resolved second-order autocorrelation in a single shot acquisition [9]].

Several schemes based on spatiotemporal characterization techniques have also been proposed to analyze the temporal structure of the pulses and the spectral dependence of the wavefront. In [3], the authors implemented a grating that images the beam into an H-S sensor, retrieving the wavefront in one axis. As to the SEA TADPOLE, it does not allow obtaining directly the wavefront of the pulse due to the instabilities of the interferometer, which introduce a phase drift from pointto-point in the spatial scan. In [10], the authors proposed the use of the iterative Gerchberg-Saxon algorithm to reduce the drift connecting by numerically propagating the spatiotemporal pattern of the pulse at multiple propagation distances.

In this paper, we study the stability of the interferometer employed in STARFISH technique [4]], demonstrating that the compact and simple configuration of the setup allows the interferometer stability required to measure the wavefront as a function of the wavelength. This method is suitable both 
for focused and not focused beams thanks to the high spatial resolution of the technique.

We apply STARFISH to retrieve the wavelength dependent wavefront of pulses focused by a kinoform diffractive lens (DL). Additionally, a full spatiotemporal characterization of the amplitude and phase evolution of such pulses in the focusing region [11] is given, obtaining a complete insight into its propagation dynamics. The numerical simulations that we performed are in excellent agreement with the experimental measurements.

In this context, the interest on the focusing properties of DL is a subject of high interest in nonlinear optics. Recently, they have been used with ultrashort laser pulses for applications such as tuning the central wavelength of the SHG [12,13] or the control of the supercontinuum generation structure and broadening [14]. These applications are based on the evolution of the spatiospectral and spatiotemporal features of the kinoform DL along the focus. Thus, the characterization of this evolution (not only on axis) is important to give deeper insight into the pulse structure involved in the different processes. The wavefront of the pulses is also analyzed in the focal region, being that this information is of huge importance for the phase-matching properties of the mentioned nonlinear processes. It is expected that, during the next years, the diffractive optical elements (DOEs) will tailor nonlinear processes such as SHG [12,13], supercontinuum [14], filamentation [15] or high-order harmonic generation [16], among others.

The possibility of measuring the wavefronts of ultrashort laser pulses as a function of their frequency content opens the way for multiple applications, e.g., the study of aberrations in optical systems [17] or the characterization of ultrashort pulses involved in nonlinear processes [13-16]. Since STARFISH has already been adapted to analyze the filamentation of light [18], it is possible to examine the wavefront dynamics in this regime to study the energy-density flux involved in the process, via the calculation of the phase (wavefront) gradient as explained in [19].

\section{EXPERIMENTAL SETUP}

The spatiotemporal characterization was carried out with STARFISH [4]. The technique consists in measuring the spatially resolved spectral interferometry by using a fiber optic coupler as interferometer. The scheme of the experimental setup is presented in Fig. 1. The reference (known) pulse is delayed to interfere with a test pulse (unknown) that is spatially scanned (using a motorized stage) with the corresponding arm of the fiber coupler. The fiber coupler arms have a length of $88 \mathrm{~cm}$. The optical system depicted in Fig. 1 is very simple, robust, and reliable and has been used before for the study of DOEs [20] and the propagation dynamics of a laser filament [16]. In our experiment, the test beam was focused by a kinoform DL, whose focus was scanned in the transverse direction to the propagation axis with a spatial resolution of $4 \mu \mathrm{m}$ (fiber mode diameter). The measurements were performed in different planes along the propagation axis, by changing the relative distance between the lens and the input fiber.

For the experiments we used a Ti:sapphire CPA laser system (Newport Corporation, USA) that delivers 100 fs pulses (Fourier-transform limited) with central wavelength of

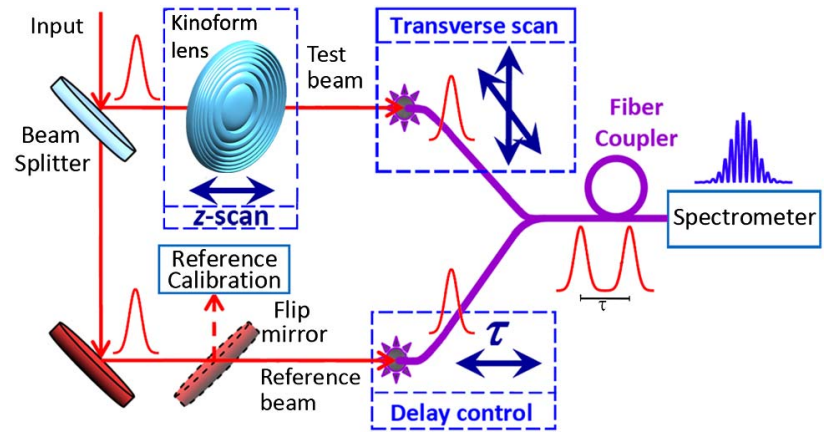

Fig. 1. (Color online) Scheme of the experimental setup: one replica of the laser pulse is used as reference and another replica is focused by a DL. The pulses are collected by the fibers of the coupler. The fiber in the reference arm controls the relative delay, whereas the fiber in the test arm spatially scans the unknown beam. The spatially resolved spectral interferometry is measured after the fiber coupler in the spectrometer. The position of the lens allows exploring different propagation distances.

$795 \mathrm{~nm}, 9 \mathrm{~nm}$ intensity full-width-half-maximum (FWHM) spectral bandwidth and $1 \mathrm{kHz}$ repetition rate. The temporal characterization of the reference pulse was performed with a GRENOUILLE device (Swamp Optics, USA) and the spectra were measured with a spectrometer of resolution $0.1 \mathrm{~nm}$ (Avantes, Netherlands). The delay between the reference and the test pulse was 2 ps.

The kinoform DL employed in the experiment (Institute of Automatics and Electrometry, Russia) has circular symmetry and a focal length given by the expression $f(\lambda)=f_{0} \lambda_{0} / \lambda$, where $\lambda$ denotes any wavelength of the pulse. The DL was designed for having a focal length of $f_{0}=150 \mathrm{~mm}$ at the wavelength $\lambda_{0}=565.1 \mathrm{~nm}$. In our case, for the central wavelength of the pulses $\lambda_{c}=795 \mathrm{~nm}$, the corresponding focal length is $f_{c} \equiv f\left(\lambda_{c}\right)=106.6 \mathrm{~mm}$. For comparison, we also employed an achromatic doublet lens (Linos G063144525) of focal length $100 \mathrm{~mm}$.

\section{EXPERIMENTAL METHOD}

In this section, we provide a description of the algorithm [21] and discuss the stability of the interferometer to be applied for wavefront sensing. For simplicity, we show the validity of the technique with the case of a focusing refractive lens. In Section 5 , the method will be applied to retrieve the complex wavefront structure at the focus of the DL.

\section{A. Amplitude and Phase Retrieval Algorithm}

In spectral interferometry, a reference (known) and a test (unknown) pulse delayed a time $\tau$ interfere in spectral domain. The resulting spectrum $S(\omega)$ can then be expressed as

$$
\begin{aligned}
S(\omega)= & S_{\text {test }}(\omega)+S_{\text {ref }}(\omega) \\
& +2 \sqrt{S_{\text {test }}(\omega) S_{\text {ref }}(\omega)} \cos \left[\phi_{\text {test }}(\omega)-\phi_{\text {ref }}(\omega)-\omega \tau\right] .
\end{aligned}
$$

The last term contains information of the phase difference between the test and the reference. By applying fringes analysis, the phase difference between the test and the reference pulses can be extracted. To do so, the spectrum is inverse Fourier transformed to the temporal domain, where it consists of three peaks. The central one at $t=0$ corresponds to the continuum contribution (i.e., the sum of both spectra), while 
the others (at $t=+\tau$ and $t=-\tau$ ) to the interference term. By filtering one of the side peaks and applying direct Fourier transform, the phase difference between the test and the reference can be extracted. Since, the reference pulse in known, the test pulse phase is then recovered [21]. The test spectrum (amplitude) can be recorded in a separate scan without the reference.

With the scheme presented in Fig. 1 this is done for different positions in the transverse plane, thus obtaining the phase difference at the measured points [we have the expression of Eq. (1) as a function of the transverse spatial coordinate]. Since cylindrical symmetry was preserved in all the cases during our study, the measurements were just performed along the $x$ axis.

By this procedure, STARFISH directly yields the spatiospectral phase of the pulses $\phi(x, \lambda)$ (for more details, please refer to [4]). Because of the numerical calculation, this phase is obtained wrapped in the range $(-\pi, \pi)$. To obtain the wavefront as a continuous function of the wavelength $\lambda$, it is only necessary to unwrap this phase.

Additionally, one should take into account that the wavefront is spatially sampled by the scan of the test beam with the fiber. Therefore, to avoid $2 \pi$ phase jumps during the experiment due to a wavefront curvature, we performed small scanning steps (1-20 $\mu \mathrm{m})$. Although the resolution of the fiber is $4 \mu \mathrm{m}$, a smaller scanning step for the focus measurement is helpful to obtain a smoother spatial profile.

\section{B. Phase Stability of the Interferometer}

It is well-known that interferometers are affected by a zeroorder phase drift, which prevents the direct measurement of the wavefront and makes necessary iterative algorithms [10]. This is also the case of our interferometer. Although in practice the zero-order phase drift does not disturb the spatiotemporal reconstructions neither the pulse front, it prevents correct wavefront retrieval because of the introduction of a phase noise.

Therefore, before using STARFISH for the wavefront measurement, we analyzed the shot-to-shot phase stability. For this purpose, we measured 20 fixed spectral interferences during $30 \mathrm{~s}$. By studying the stability during $30 \mathrm{~s}$ (a time larger than the typical time of a measurement), we ensure that the measurements are not affected by other instabilities sources at larger time scales than the scan itself. We studied the evolution of the zero-order phase drift: the maximum phase drift was $0.35 \cdot 2 \pi$ radian and the standard deviation $0.09 \cdot 2 \pi$ radian. This phase drift is low enough to directly measure the wavefronts with an error given by this phase drift.

The phase drift fluctuation is originated mainly by air-flow fluctuations in the free-space portion of the setup or by vibrations. In our case, the error is small enough to measure the pulses. However, these fluctuations could be minimized by reducing the air-flow in the setup, in the case of an experiment that required more precision.

In general, the duration of a spatial scan will depend on the number of sampling points and the integration time. A typical scan-around 100 points and $2 \mathrm{~ms}$ integration time-takes less than $1 \mathrm{~min}$. In the case of 2D spatial scans ( $x, y$ axes), this would require more sampling points and put more demand on the phase stability.

\section{Proof of Concept}

To discuss the validity of STARFISH as wavefront sensor for the characterization of DOEs, we computed the wavefront for the convergent beam presented in the Fig. 4 of [4] (Subsection 3.C). In that case, a system delivering 35 fs pulses (intensity FWHM) with a central wavelength of $795 \mathrm{~nm}$ and $10 \mathrm{~Hz}$ repetition rate was employed for the experiment. The test beam was focused with a refractive lens with a focal length of $50 \mathrm{~cm}$ and the measurement was performed $31 \mathrm{~cm}$ after this lens. The spatial profile of the pulse was transversely scanned in one direction with steps of $20 \mu \mathrm{m}$ across $4 \mathrm{~mm}$. Using the data measured in that experiment, we retrieved the wavefront in one axis $\phi\left(x ; \lambda=\lambda_{j}\right)$ for different wavelengths $\lambda_{j}$ of the pulse spectrum [see Fig. 2(a)]. Note that, to depict each wavelength a different color has been used. The expected phase is quadratic, given by the expression $\phi\left(x ; \lambda=\lambda_{j}\right)=$ $-\left(\pi / \lambda_{j} R\right) x^{2}$, where $R$ denotes the radius of curvature. In agreement with the experiment, the shorter wavelengths correspond to the higher curvatures. Then, we calculated the coefficient of the quadratic term of the phase for each wavelength defined as $\kappa\left(\lambda_{j}\right)=-\pi / \lambda_{j} R$ [see blue solid line in Fig. 2(b)]. From the linear regression of the data we obtained a value of $R=-189.8 \pm 1.7 \mathrm{~mm}$, also in good agreement with the expected value $(R=-190 \mathrm{~mm})$. The fit is represented as a dashed red line in Fig. 2(b), where the gray shaded area stands for the uncertainty of the fit.

\section{THEORETICAL MODEL FOR THE NUMERICAL SIMULATIONS}

In order to predict the focusing dynamics of the pulses after the DL and to validate the experimental measurements, we simulated numerically the propagation of such pulses. For this purpose, we calculated the electric field diffracted by the DL using the theory of Fresnel's diffraction. To model the lens, one should take into account the kinoform profile of the lens as detailed in [11]. However, this exact approach requires a very computationally demanding sampling in the spatial domain. Instead, for the calculations one can model the effect of the DL as the phase introduced by a thin lens with focal variable given by the expression $f(\lambda)=f_{0} \lambda_{0} / \lambda$.

To calculate the diffracted electric field $U_{2}$ at the focus from the electric field $U_{1}$ that illuminates the DL, we take advantage of the cylindrical symmetry of the system and express them as a function of the radial coordinates $r_{1}$ and $r_{2}$ in the
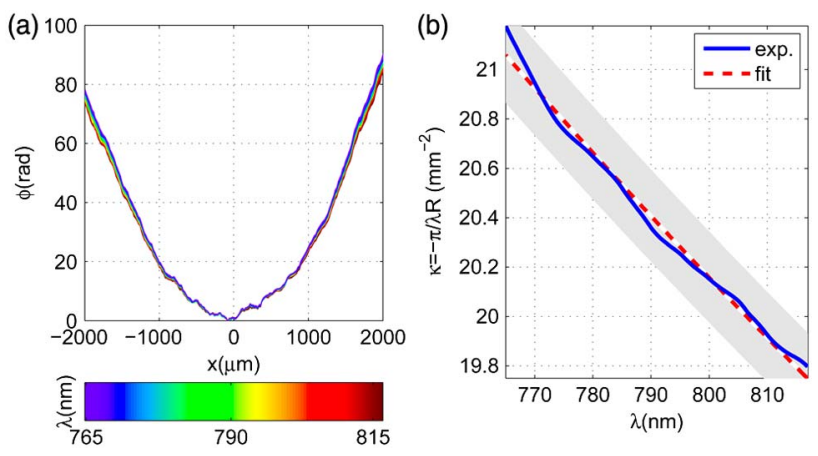

Fig. 2. (Color online) (a) Wavefront as a function of the wavelength for a convergent wave (focused by a refractive lens). The wavefront for each wavelength is plotted in the color given by the colorbar; (b) Curvature of wavefronts (solid blue line) and theoretical value (dashed red line). 
input and output planes, respectively. Under the paraxial approximation, the Fresnel's diffraction can be expressed as follows [22]:

$$
\begin{aligned}
U_{2}\left(r_{2}, \lambda\right)= & i \frac{2 \pi}{\lambda z} \exp \left(-\frac{i k r_{2}^{2}}{2 z}\right) \\
& \times \int_{0}^{\infty} U_{1}\left(r_{1}, \lambda\right) \exp \left(-\frac{i k r_{1}^{2}}{2 z}\right) J_{0}\left(\frac{k r_{1} r_{2}}{z}\right) r_{1} \mathrm{~d} r_{1},
\end{aligned}
$$

where $\lambda$ is the wavelength, $z$ is the propagation distance, $k$ is the wave vector, and $J_{0}$ is the Bessel function of the first kind of zero order. The input electric field $U_{1}$ in spatial domain is a Gaussian profile with a full-width at $1 / e^{2}$ in intensity of $7 \mathrm{~mm}$, which agrees very well with the experimental conditions. In the same way, the experimental spectral amplitude was also included (FWHM of $9 \mathrm{~nm}$ in intensity). Finally, to model the DL, we introduced it as the phase of a lens of variable focal $\exp \left\{i k r^{2} / 2 f(\lambda)\right\}$.

Therefore, we calculate the spatiospectral amplitude and phase of the electric field $U_{2}\left(r_{2}, \lambda\right)$ for different propagation distances $z$ in the vicinity of the focus using Eq. (2). Then, we obtain the corresponding amplitude and phase in spatiotemporal domain by inverse Fourier-transform.

\section{EXPERIMENTAL MEASUREMENTS AND COMPARISON WITH SIMULATIONS}

\section{A. Measurement of the Focus of an Achromatic Lens}

We first measured with STARFISH the pulse focused with the achromatic doublet $(f=100 \mathrm{~mm})$, in order to compare the focusing characteristics of the DL with that of the achromatic doublet. The experimental results are shown in Fig. 3. In this case, we can assume that the focal length does not depend on the wavelength. Therefore, the spatially resolved spectrum [Fig. 3(a)] shows a spatial width almost independent on the wavelength. The spatiotemporal intensity [Fig. 3(b)] corresponds to an undistorted focused pulse. The results on axis $(x=0)$ show a nondistorted spectrum of $9 \mathrm{~nm}$ FWHM [Fig. 3(c)] similar to the input one, which in the temporal domain corresponds to a Gaussian pulse with duration comparable to the input pulse [Fig. 3(d)]. The intensity profile is colored by the instantaneous wavelength of the pulse. The
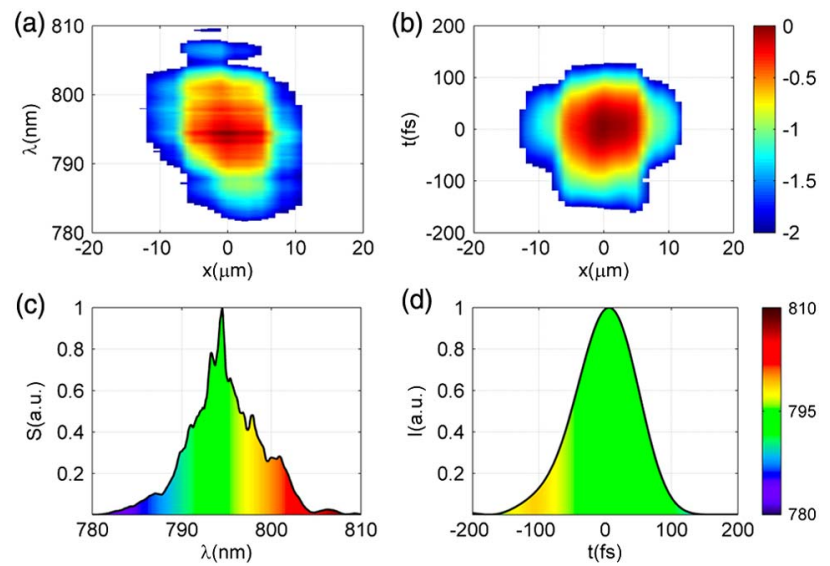

Fig. 3. (Color online) Results of the characterization at the focus of the achromatic doublet lens: (a) Spatially resolved spectrum; (b) Spatiotemporal intensity; (c) Spectrum on axis; (d) Intensity on axis colored by the instantaneous wavelength. The plots (a) and (b) are in a logarithmic scale (see colorbar) that comprises 2 orders of magnitude. instantaneous wavelength is obtained as the inverse of the instantaneous frequency calculated as the derivative of the temporal phase of the pulse, so it gives the information of the pulse temporal chirp. As it can be seen, the on-axis instantaneous wavelength profile is very flat.

\section{B. Evolution of the Wavefront and the Spectrum with a DL}

To track the evolution of the pulse around the focus of the DL $\left(f_{c}\right)$ and test influence of the phase shift on the measurement, we measured the full spatiotemporal amplitude and phase at the following propagation distances $(z): f_{c}-2 \mathrm{~mm}, f_{c}-1 \mathrm{~mm}$, $f_{c}, f_{c}+1 \mathrm{~mm}$ and $f_{c}+2 \mathrm{~mm}\left(f_{c}=106.6 \mathrm{~mm}\right)$. For the three central positions, we scanned $100 \mu \mathrm{m}$ of the transverse profile with $1 \mu \mathrm{m}$ steps. For the two external positions, we scanned $200 \mu \mathrm{m}$ of the transverse profile in $2 \mu \mathrm{m}$ steps.

As it was explained before, we measured the spatiospectral phase with STARFISH, which gives us the wavefront resolved in frequencies. In Fig. 4, we have plotted the lineouts of the wavefronts $\phi\left(x ; \lambda_{j}\right)$ for the different wavelengths $\lambda_{j}$ of the pulse spectrum. Each wavelength corresponds to a color given by the colorbar inset in Fig. 4 . The results are presented for the propagation distances labeled in the left. The left column shows the simulation, whereas the right column shows the experimental results. The resulted wavefronts clearly show the chromatic dependence due to the focal length $f(\lambda)$. For the first position, $z_{-2}=f_{c}-2 \mathrm{~mm}$, the reddish wavelength $\sim 808 \mathrm{~nm}$ is just arriving its focus, so all the wavefronts have positive curvature (converging). As expected for a Gaussian beam around the focus, the higher curvature corresponds to the bluer wavelengths. At longer propagation distances, the focus moves toward shorter wavelengths. For instance, at $z_{-1}=f_{c}-1 \mathrm{~mm}$, the wavelength $\sim 802 \mathrm{~nm}$ is focused: longer wavelengths are after the focus, thus showing negative curvature (diverging), while the remaining wavelengths are still focusing (converging). At the position of the focus for the central wavelength $795 \mathrm{~nm}$, i.e., $z_{0}=f_{c}$ (mm), half of the spectrum is before the focus (the bluer), while the other half (the reddish) is after the focus. This yields positive and negative wavefront curvatures, respectively. As we move apart from the distance $z_{0}$, the shorter wavelengths are focused. For instance, at $z_{+1}=f_{c}+1 \mathrm{~mm}$, the focusing wavelength corresponds to $\sim 786 \mathrm{~nm}$. At the last position, $z_{+2}=f_{c}+2 \mathrm{~mm}$, the bluer wavelength $\sim 781 \mathrm{~nm}$ is focused and the whole pulse is after the focus, exhibiting negative curvature in the wavefront (thus diverging).

The agreement between the experiment and the theory is quite good, presenting a small effect of the phase drift error. Notice that this error affects the central positions more because the curvature is smaller and thus comparable with the error. Also, the retrieved wavefront is noisy and not reliable for the wavelengths where the spectral amplitude is small and the signal is too low (see the corresponding Fig. 5). Finally, the slight spatial asymmetry (x axis) in the experimental data shown in Fig. 4 could be originated by a misalignment of the setup, in particular in the case of a not perfect scan perpendicular to the $z$-axis.

The simulated and experimental spatially resolved spectrum as a function of the propagation distance is depicted in Fig. 5. The real pulse spectrum has been taken into account 

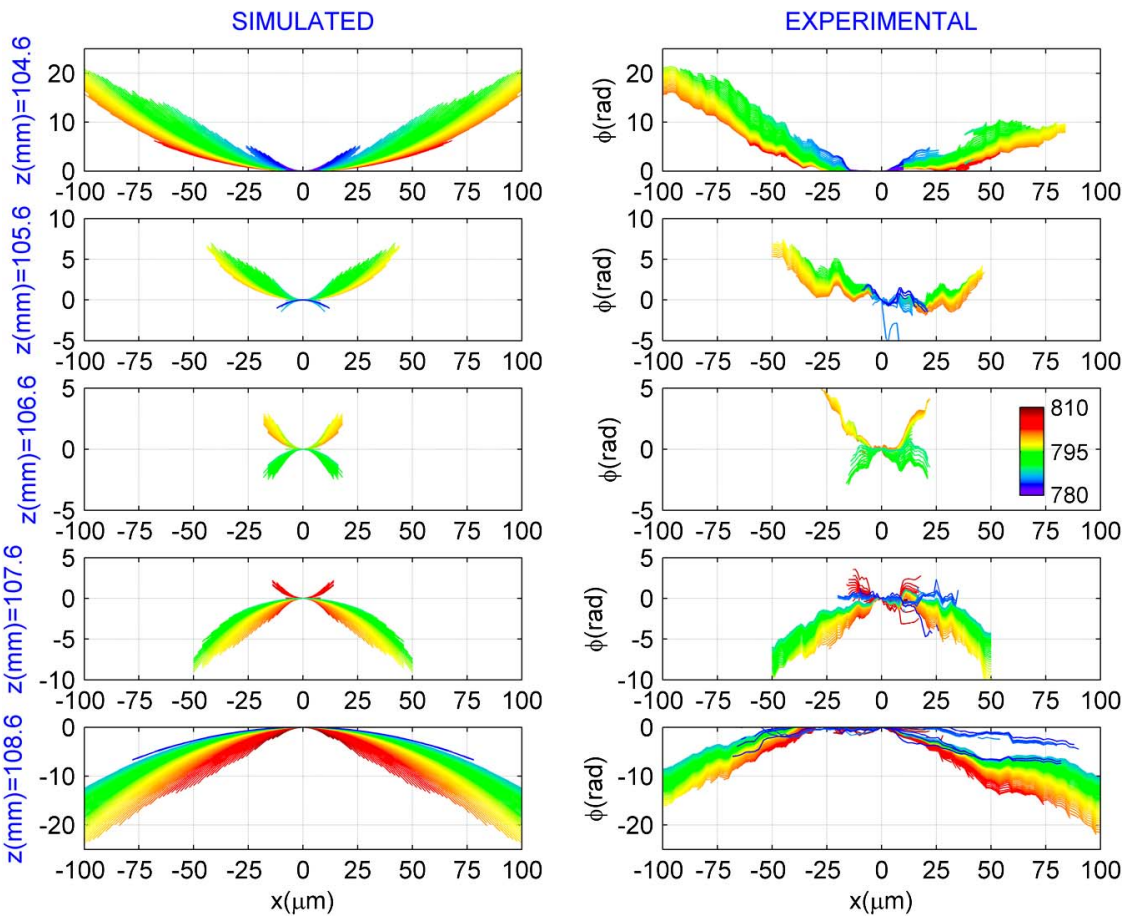

Fig. 4. (Color online) Simulated (left) and experimental (right): wavefront as a function of the wavelength before and after the focus of the DL. The wavefront for each wavelength is plotted in the color given by the colorbar inset (the same colorbar applies to all subplots in the figure).

in the simulations, which allows us to achieve a very good agreement between the theory and the experiment. We observe for the spectrum's amplitude the same behavior described for the wavefronts. The results of the spatial scan show how the spatial width for each wavelength varies with the propagation distance. This allows us to easily identify the wavelengths that are before and after the focus as a function of the propagation distance. For $z_{-2}$, the reddish wavelength
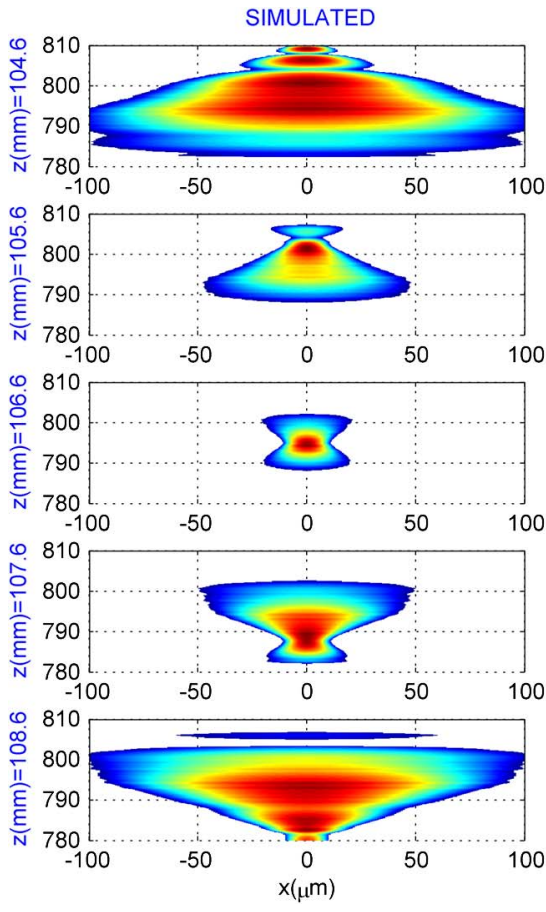

again is just at its focus position. For $z_{-1}$, the reddish wavelengths are after focus, whereas for $z_{0}$ the central wavelength is focused and the focus is symmetric. After the focus, for $z_{+1}$ and $z_{+2}$, the reddish wavelengths are diverging and the bluer ones reach the focus and then start to diverge. The combined analysis of the amplitude and the wavefront of the spectrum made possible to analyze the dependence of the focusing process on the wavelengths.
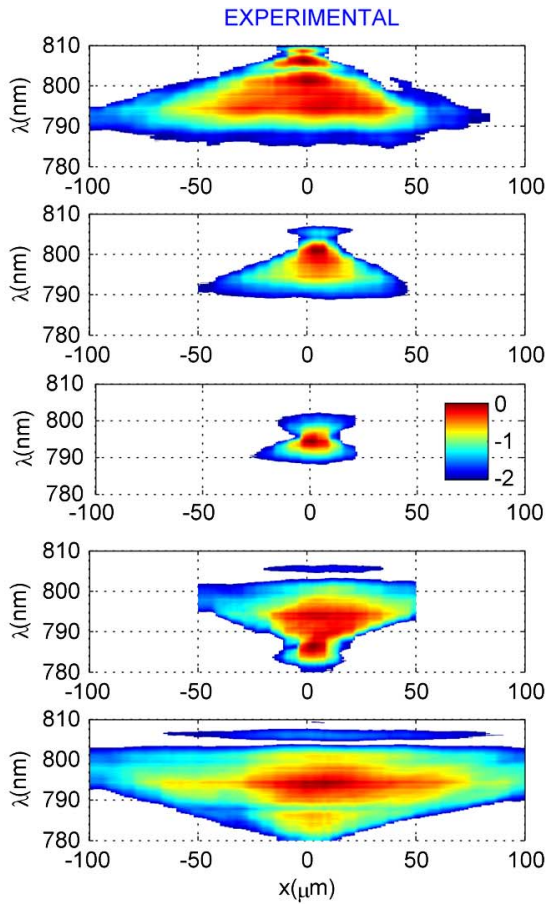

Fig. 5. (Color online) Simulated (left) and experimental (right): spatially resolved spectrum before and after the focus of the DL. The logarithmic scale comprises 2 orders of magnitude (see colorbar inset). 

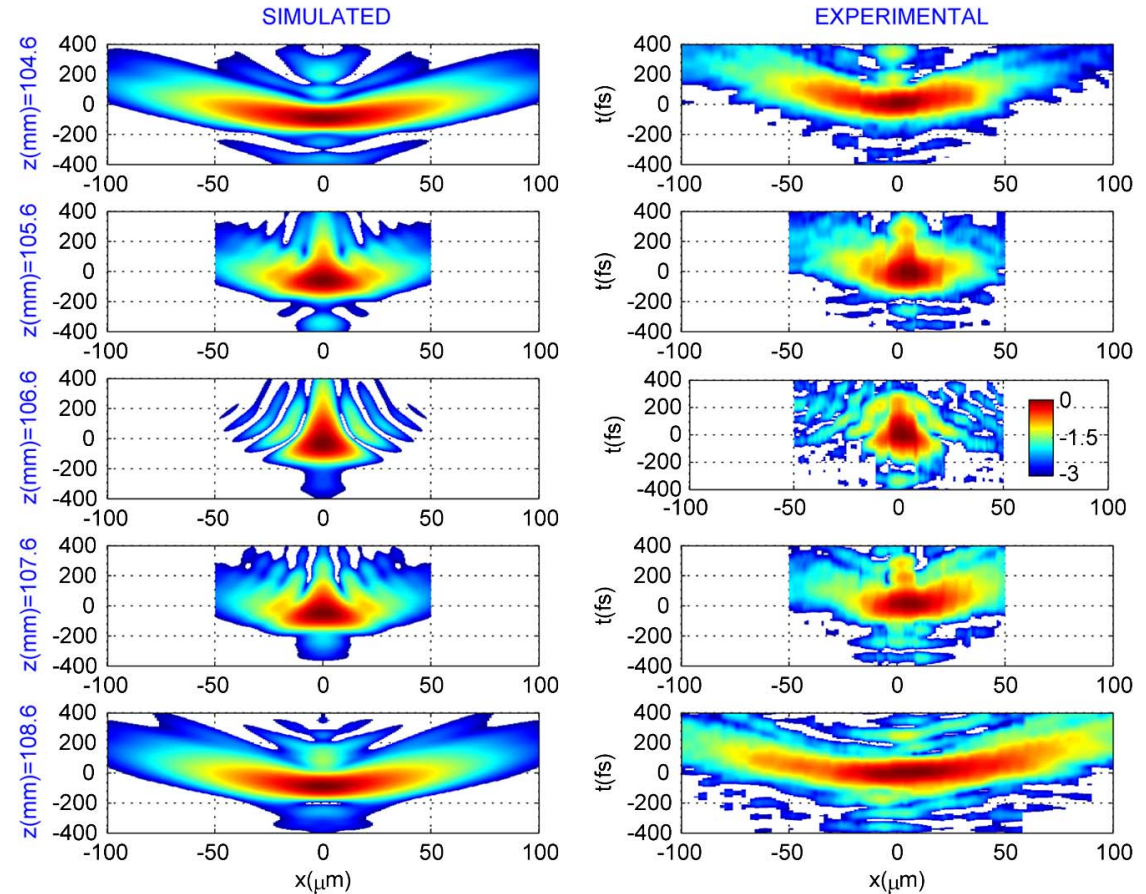

Fig. 6. (Color online) Simulated (left) and experimental (right): spatiotemporal intensity before and after the focus of the DL. The logarithmic scale comprises 3 orders of magnitude (see colorbar inset).

\section{Spatiotemporal Dynamics}

To gain a deeper insight into the dynamics of the processes involved in the focusing of ultrashort pulses by a DL, a complete spatiotemporal study is mandatory. In such cases, it is not enough to know the structure of the pulse on axis because the whole spatial profile is involved in the process. The spatiotemporal reconstructions made by STARFISH are in very good agreement with the simulations, which are shown for different propagation distances in Fig. 6 .

We observe that the pulse front curvature is the same at both sides of the focus. The measurements of the wavefront (phase front) and the pulse front are in agreement with the
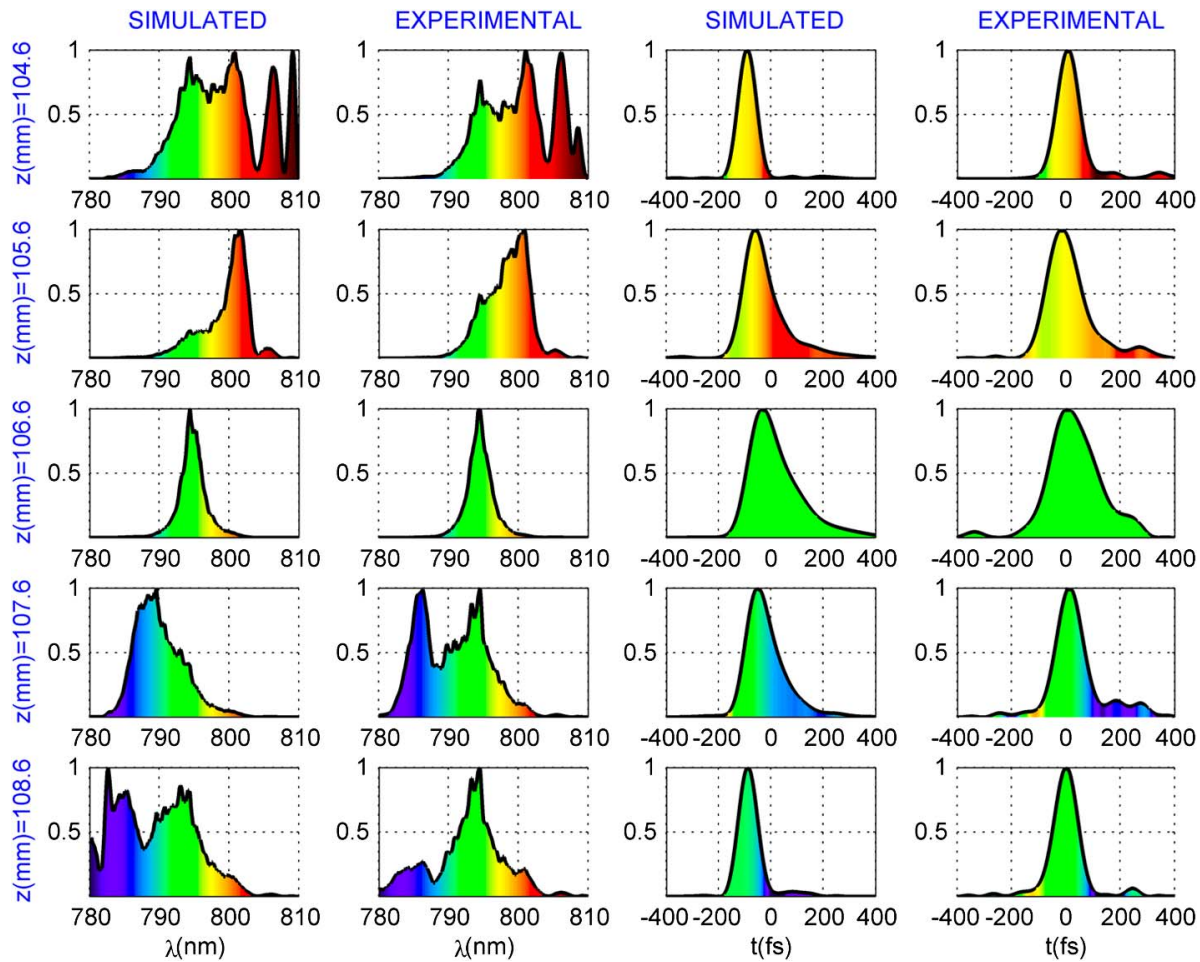

Fig. 7. (Color online) On-axis normalized simulated spectrum (first column) and experimental spectrum (second column), simulated intensity (third column) and experimental intensity (forth column), as a function of the propagation distance (see labels on the left). The spectra are colored by their wavelengths. The same color scale applies to represent the instantaneous wavelength in the temporal intensity plots. 
predictions presented in the work [23] for chromatic elements. In that work, Bor found out that the wavefront is symmetric with respect to the focus, being flat for the central wavelength at its focus. Regarding the curvature of the pulse front, it is the same as that of a divergent wave before and after the focus, being flatter after the focus. For the focus position $\left(z=z_{0}\right)$, the spatiotemporal intensity corresponds to the far-field structure, already observed in [20], with a main broadened central peak, and a train of pulses in the wings coming from the ring structure.

The slight asymmetry respect to the focus $\left(z=f_{c}\right)$ observed in the spatiotemporal intensity patterns is only due to the nonsymmetric input spectrum. To ensure this, we did the simulations using a 9 nm FWHM Gaussian input spectrum and obtained symmetric patterns.

\section{Temporal and Spectral Results on Axis}

To complete the analysis, it is very helpful to observe directly the pulse on axis in the spectral and temporal domains. The simulated and experimental results are shown in Fig. 7 for the different propagation distances. The information of Fig. 7 is completed with Table 1 , where we give the values associated with the pulses (both simulated and experimental): gravity center of the spectrum, instantaneous wavelength of the pulse at its maximum and FWHM in temporal domain.

The effect of the variable focal length makes the spectrum narrower in the focus $\left(z=z_{0}\right)$, which corresponds to a longer pulse on axis with an instantaneous wavelength close to the central wavelength of the pulse. This narrowing in the spectrum comes from the fact that the other wavelengths are out of focus, yielding a higher ratio between the spectral intensity of the central wavelength and that of the remaining wavelengths. In contrast, out of the nominal focus $f_{c}$, the tails of the spectrum are enhanced, which corresponds to a broader (flatter) spectrum and therefore shorter pulses on axis. Similar results are obtained for the experiments and the simulations.

\section{E. Comparison of the Results at Different Levels of Intensity}

Taking advantage of the whole information obtained with STARFISH and the cylindrical symmetry of the system, it is possible to depict a representation of the amplitude and phase of the pulse in spatiotemporal domain. The amplitude is represented as an iso-intensity surface, i.e., $I(x, y, t)=\alpha \cdot I_{\max }$ (being $\alpha$ a constant), where cylindrical symmetry has been assumed to extend the results to the spatial variable $y$. The phase is represented by coloring with the instantaneous wavelength evaluated at the surface $I(x, y, t)$. Each instantaneous wavelength is represented by a color. In Fig. $\underline{8}$, the

Table 1. Spectral and Temporal Parameters of the Pulse Measured on Axis

\begin{tabular}{|c|c|c|c|c|c|c|}
\hline \multirow[b]{2}{*}{$z(\mathrm{~mm})$} & \multicolumn{2}{|c|}{ Gravity Center (nm) } & \multicolumn{2}{|c|}{$\lambda_{\text {inst }}(\mathrm{nm})^{a}$} & \multicolumn{2}{|c|}{ Time FWHM (fs) } \\
\hline & Simul. & Exper. & Simul. & Exper. & Simul. & Exper. \\
\hline 104.6 & 799.2 & 800.0 & 797.9 & 798.5 & 82.6 & 96.0 \\
\hline 105.6 & 799.4 & 798.3 & 798.0 & 797.1 & 122.4 & 141.3 \\
\hline 106.6 & 794.8 & 794.5 & 794.8 & 794.2 & 165.4 & 187.4 \\
\hline 107.6 & 790.1 & 790.7 & 790.8 & 791.9 & 129.4 & 113.6 \\
\hline 108.6 & 789.5 & 792.8 & 790.7 & 793.1 & 85.5 & 100.7 \\
\hline
\end{tabular}

$\lambda_{\text {inst }}$ stands for the instantaneous wavelength of the pulse at its maximum
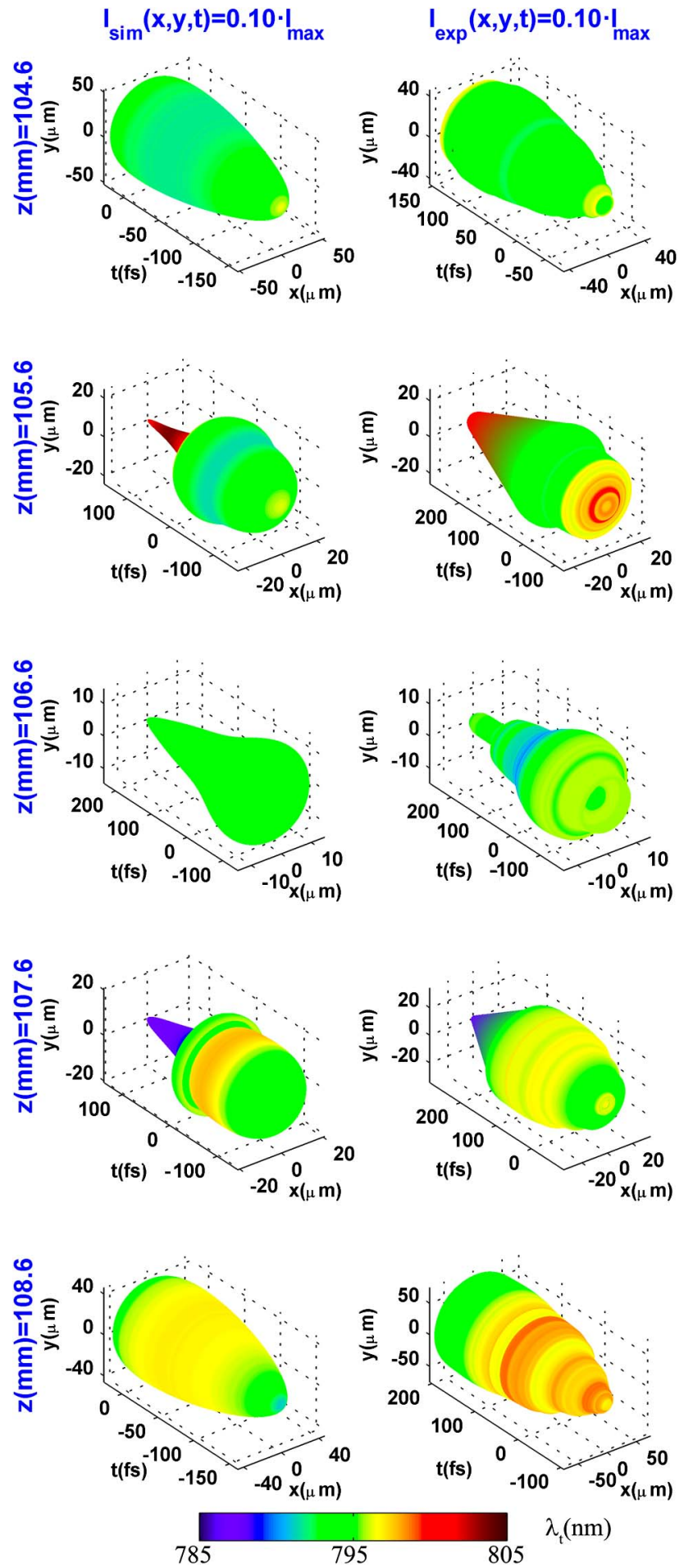

Fig. 8. (Color online) Iso-intensity surfaces $I(x, y, t)=\alpha \cdot \operatorname{Imax}-$ colored by the corresponding instantaneous wavelength-before and after the focus of the DL. The simulated (left) and experimental (right) results for $\alpha=0.1$ are shown. Cylindrical symmetry is assumed to obtain the plot. Media 1 shows the same results for different levels of $\alpha$ ranging from 0.01 to 0.9 .

simulated and experimental results for the different propagation distances are compared for the value $\alpha=0.1$. The same plot has been done for different levels of the surface, as it is shown in the animation of Media 1. This information gives an idea of the pulse structure and their chirp at different levels. 


\section{CONCLUSION}

In this work, we have demonstrated the capability of STARFISH to characterize the wavefront of the pulses. This wavefront reconstruction allows resolution of the wavelength dependence, which is of high interest in many situations, as highlighted in the introduction. In contrast to standard wavefront sensing techniques, our high spatial resolution allows applying STARFISH to focusing beams. We should also emphasize here that STARFISH retrieves the spatiotemporal and spatiospectral amplitude and phases, being a complete tool for the experimental description of the pulse dynamics. Notice that this full amplitude and phase characterization allows simulation of the subsequent propagation of the pulses through an optical system or evaluating their interaction in a certain process. We found that there is an intrinsic phase drift error in the measurements, but its low value $(0.35 \cdot 2 \pi)$ allows us to reconstruct the wavefronts of the pulses.

We have applied the method for the characterization of DOEs, which is a topic of high interest nowadays. The wavefront retrieval and the complete study of the pulse dynamics in the focusing region of a kinoform DL were performed. In this context, we studied the frequency resolved wavefronts in the vicinity of the focus, which are responsible for the phasematching in many nonlinear processes. The spatiotemporal, spatiospectral, and on-axis results are also helpful to understand these processes, e.g., the SHG tuning, explained by the spectral waveform modulation due to the focal length dependent on wavelength. In the time domain, the on-axis pulse is longer at the focus because of the spectral narrowing, whereas due to the opposite effect it is shortened before and after focus.

The experimental results presented in this paper were compared with numerical simulations of the diffracted electric field, presenting an excellent agreement.

\section{ACKNOWLEDGMENTS}

We acknowledge support from Spanish Ministerio de Ciencia e Innovación (MICINN) through the Consolider Program SAUUL (CSD2007-00013), Research projects FIS2009-09522, FIS2010-15746 and P11B2010-26, and grant programs Formación de Profesorado Universitario (for B. Alonso and R. Borrego-Varillas) and Ramón y Cajal (for I. J. Sola); and from the Junta de Castilla y León through the Program for Groups of Excellence (GR27).

\section{REFERENCES}

1. I. A. Walmsley and C. Dorrer, "Characterization of ultrashort electromagnetic pulses,” Adv. Opt. Photon. 1, 308-437 (2009).

2. P. Bowlan, P. Gabolde, and R. Trebino, "Directly measuring the spatiotemporal electric field of focusing ultrashort pulses," Opt. Express 15, 10219-10230 (2007).

3. E. Rubino, D. Faccio, L. Tartara, P. K. Bates, O. Chalus, M. Clerici, F. Bonaretti, J. Biegert, and P. Di Trapani, "Spatiotemporal amplitude and phase retrieval of space-time coupled ultrashort pulses using the Shackled-FROG technique," Opt. Lett. 34, 3854-3856 (2009).

4. B. Alonso, I. J. Sola, O. Varela, J. Hernández-Toro, C. Méndez, J. San Román, A. Zaïr, and L. Roso, "Spatiotemporal amplitude- and-phase reconstruction by Fourier-transform of interference spectra of high-complex-beams," J. Opt. Soc. Am. B 27, 933-940 (2010).

5. R. V. Shack and B. C. Platt, "Production and use of a lenticular Hartmann screen," J. Opt. Soc. Am. 61, 656-660 (1971).

6. S. Velghe, J. Primot, N. Guérineau, M. Cohen, and B. Wattellier, "Wave-front reconstruction from multi-directional phase derivatives generated by multilateral shearing interferometers," Opt Lett. 30, 245-247 (2005).

7. C. P. Hauri, J. Biegert, U. Keller, B. Schaefer, K. Mann, and G. Marowski, "Validity of wave-front reconstruction and propagation of ultrabroadband pulses measured with a Hartmann-Shack sensor," Opt. Lett. 30, 1563-1565 (2005).

8. R. Borrego-Varillas, C. Romero, J. R. Vázquez de Aldana, J. M. Bueno, and L. Roso, "Wavefront retrieval of amplified femtosecond beams by second-harmonic generation," Opt. Express 19, 22851-22862 (2011).

9. R. Grunwald, U. Neumann, U. Griebner, K. Reimann, G. Steinmeyer, and V. Kebbel, "Ultrashort-pulse wave-front autocorrelation," Opt. Lett. 28, 2399-2401 (2003).

10. P. Bowlan and R. Trebino, "Using phase diversity for the measurement of the complete spatiotemporal electric field of ultrashort laser pulses," J. Opt. Soc. Am. B 29, 244-248 (2012).

11. V. Moreno, J. F. Román, and J. R. Salgueiro, "High efficiency diffractive lenses: deduction of kinoform profile,” Am. J. Phys. 65, 556-562 (1997).

12. G. Mínguez-Vega, C. Romero, O. Mendoza-Yero, J. R. Vázquez de Aldana, R. Borrego-Varillas, C. Méndez, P. Andrés, J. Lancis, V. Climent, and L. Roso, "Wavelength tuning of femtosecond pulses generated in nonlinear crystals by using diffractive lenses," Opt. Lett. 35, 3694-3696 (2010).

13. C. Yang, K. Shi, H. Li, Q. Xu, V. Gopalan, and Z. Liu, "Chromatic second harmonic imaging," Opt. Express 18, 23837-23843 (2010).

14. C. Romero, R. Borrego-Varillas, A. Camino, G. Mínguez-Vega, O. Mendoza-Yero, J. Hernández-Toro, and J. R. Vázquez de Aldana, "Diffractive optics for spectral control of the supercontinuum generated in sapphire with femtosecond pulses," Opt. Express 19, 4977-4984 (2011).

15. Y. Fu, H. Xiong, H. Xu, J. Yao, B. Zeng, W. Chu, Y. Cheng, Z. Xu W. Liu, and S. L. Chin, "Generation of extended filaments of femtosecond pulses in air by use of a single-step phase plate," Opt. Lett. 34, 3752-3754 (2009).

16. L. Van Dao, K. Ba Dinh, and P. Hannaford, "Generation of extreme ultraviolet radiation with a Bessel-Gaussian beam," Appl. Phys. Lett. 95, 131114 (2009).

17. J. Jasapara and W. Rudolph, "Characterization of sub-10 fs pulse focusing with high-numerical-aperture microscope objectives," Opt. Lett. 24, 777-779 (1999).

18. B. Alonso, I. J. Sola, J. San Román, Ó. Varela, and L. Roso, "Spatiotemporal evolution of light during propagation in filamentation regime," J. Opt. Soc. Am. B 28, 1807-1816 (2011).

19. D. Faccio, A. Lotti, A. Matijosius, F. Bragheri, V. Degiorgio, A Couairon, and P. Di Trapani, "Experimental energy-density flux characterization of ultrashort laser pulse filaments," Opt. Express 17, 8193-8200 (2009).

20. O. Mendoza-Yero, B. Alonso, O. Varela, G. Mínguez-Vega, Í. J. Sola, J. Lancis, V. Climent, and L. Roso, "Spatio-temporal characterization of ultrashort pulses diffracted by circularly symmetric hard-edge apertures: theory and experiment," Opt. Express 18, 20900-20911 (2010).

21. L. Lepetit, G. Cheriaux, and M. Joffre, "Linear techniques of phase measurement by femtosecond spectral interferometry for applications in spectroscopy," J. Opt. Soc. Am. B 12, 2467-2474 (1995).

22. M. Gu, Advanced Optical Imaging Theory (Springer-Verlag, 1999).

23. Z. Bor, "Distortion of femtosecond laser pulses in lenses," Opt Lett. 14, 119-121 (1989). 